\title{
Building Blocks of the Milky Way's Stellar Halo
}

\section{Pim van Oirschot ${ }^{1}$, Else Starkenburg ${ }^{2}$, Amina Helmi ${ }^{3}$ and Gijs Nelemans ${ }^{1,4}$}

\author{
${ }^{1}$ Department of Astrophysics/IMAPP, Radboud University Nijmegen, \\ P.O. Box 9010, 6500 GL Nijmegen, The Netherlands, email: P.vanOirschot@astro.ru.nl \\ ${ }^{2}$ Leibniz-Institut fur Astrophysik Potsdam, An der Sternwarte 16, D-14482 Potsdam, Germany \\ ${ }^{3}$ Kapteyn Astronomical Institute, University of Groningen, \\ P.O. Box 800, 9700 AV, Groningen, The Netherlands \\ ${ }^{4}$ Institute for Astronomy, KU Leuven, Celestijnenlaan 200D, 3001 Leuven, Belgium
}

\begin{abstract}
We study the assembly history of the stellar halo of Milky Way-like galaxies using the six high-resolution Aquarius dark matter simulations combined with the Munich-Groningen semi-analytic galaxy formation model. Our goal is to understand the stellar population contents of the building blocks of the Milky Way halo, including their star formation histories and chemical evolution, as well as their internal dynamical properties. We are also interested in how they relate or are different from the surviving satellite population. Finally, we will use our models to compare to observations of halo stars in an attempt to reconstruct the assembly history of the Milky Way's stellar halo itself.
\end{abstract}

Keywords. Galaxy: halo, Galaxy: evolution

We post-process the Aquarius simulations (Springel et al. 2008) with the MunichGroningen semi-analytic galaxy formation model (Starkenburg et al. 2013, and references therein) to model six Milky Way-like galaxies (A-F). The stellar masses of the spheroids (stellar halos including the inner few $\mathrm{kpc}$ bulge region) of these galaxies are ranging from $5 \cdot 10^{9}-2 \cdot 10^{10} M_{\odot}$, which are typically more massive than the analogous stellar halos in the GALFORM semi-analytic model (Cooper et al. 2010).

In total we find approximately 100 building block galaxies per spheroid, many of which however contribute only a small amount of halo stars. In agreement with Cooper et al. (2010), we find that the stellar halo is built up mainly by a few main progenitor galaxies. In Figure 1 we show the Age-Metallicity maps of eight building blocks of halo $\mathrm{C}$ compared with the Age-Metallicity maps of eight surviving satellites of that halo of similar stellar mass. The main progenitor building blocks are visualized in the upper four panels. Comparing these panels with the four in the second row, we see that the $[\mathrm{Mg} / \mathrm{H}]$ values for these two classes of objects are very comparable (approximately solar) but the building blocks stop forming stars earlier on average. In the third and fourth row, we plot Age-Metallicity maps of $\sim 10^{5} M_{\odot}$ building blocks and surviving satellites respectively. These smaller mass systems are clearly different from the more massive systems in that they stop their star formation at earlier times. Furthermore, these bottom two rows look more alike.

We plan to do a statistical analysis on the differences between the building blocks and the surviving satellites for all the halos, looking at the Mass-Metallicity relations and average star formation rates amongst others. We will compare our findings to results in 


\section{Comparison building blocks and satellites halo $\mathrm{C}$}

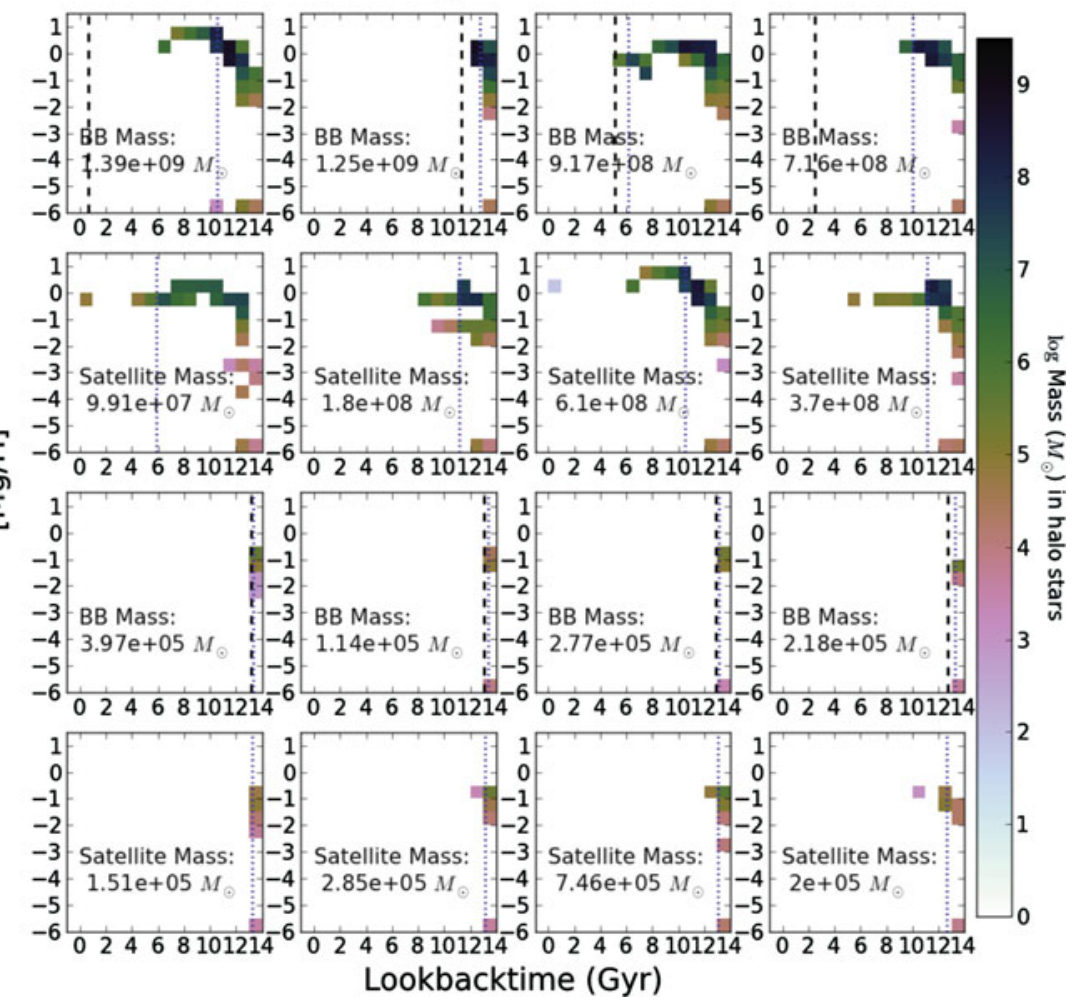

Figure 1. Age-Metallicity maps of building blocks of halo C (first and third row) compared with those of surviving satellites (second and fourth row) of that halo of similar stellar mass. The blue dotted line in each panel represents the time of infall (when the galaxy became a satellite), the black dashed lines in the building block panels indicate the time of merging with the main halo (see Starkenburg et al. 2013, how exactly this is defined).

the literature (eg. Font et al. 2006). Furthermore, we plan to couple a binary population synthesis code to this semi-analytic galaxy formation model in order to study binary evolution in a cosmological context. Doing this, one obtains inputs for the population synthesis modelling from the galaxy formation model such as a realistic star formation rate on the one hand, and inputs for the semi-analytic galaxy formation model from the population synthesis code such as yields from Supernovae (see also Yates et al. 2013) on the other hand.

\section{References}

Springel et al. 2008, MNRAS, 391, 1685

Starkenburg et al. 2013, MNRAS, 429, 725

Cooper et al. 2013, MNRAS, 406, 744

Font et al. 2006, ApJ, 638, 585

Yates et al. 2013, MNRAS, 435, 3500 